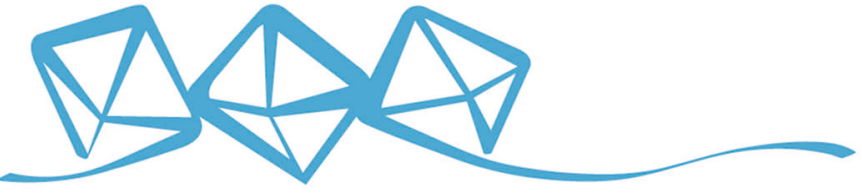 COMMUNICATIONS MATERIALS
}

ARTICLE

https://doi.org/10.1038/s43246-020-00070-6

OPEN

\section{Dynamic observation of manganese adatom mobility at perovskite oxide catalyst interfaces with water}

\author{
Gaurav Lole ${ }^{1,2}$, Vladimir Roddatis ${ }^{1,7}$, Ulrich Ross (1) 1, Marcel Risch (1) 1,3, Tobias Meyer (1) 4, Lukas Rump (1) 4,5, \\ Janis Geppert (10) 1, Garlef Wartner (10) 1,8, Peter Blöchl (1) ${ }^{5,6}$ \& Christian Jooss (i) 1,2凶
}

Real time in-situ microscopy imaging of surface structure and atom dynamics of heterogeneous catalysts is an important step for understanding reaction mechanisms. Here, using in-situ environmental transmission electron microscopy (ETEM), we directly visualize surface atom dynamics at manganite perovskite catalyst surfaces for oxygen evolution reaction (OER), which are $\geq 20$ times faster in water than in other ambients. Comparing (001) surfaces of $\mathrm{La}_{0.6} \mathrm{Sr}_{0.4} \mathrm{MnO}_{3}$ and $\operatorname{Pr}_{0.67} \mathrm{Ca}_{0.33} \mathrm{MnO}_{3}$ with similar initial manganese valence state and OER activity, but very different OER stability, allows us to distinguish between reversible surface adatom dynamics and irreversible surface defect chemical reactions. We observe enhanced reversible manganese adatom dynamics due to partial solvation in adsorbed water for the highly active and stable $\mathrm{La}_{0.6} \mathrm{Sr}_{0.4} \mathrm{MnO}_{3}$ system, suggesting that aspects of homogeneous catalysis must be included for understanding the OER mechanism in heterogeneous catalysis.

\footnotetext{
${ }^{1}$ Institute of Materials Physics, University of Goettingen, Friedrich-Hund-Platz 1, 37077 Goettingen, Germany. ${ }^{2}$ International Center for Advanced Studies of Energy Conversion (ICASEC), University of Goettingen, D-37077 Goettingen, Germany. ${ }^{3}$ Helmholtz-Zentrum Berlin für Materialien und Energie GmbH, Young Investigator Group Oxygen Evolution Mechanism Engineering, 14109 Berlin, Germany. ${ }^{4}$ 4th Physical Institute, Solids and Nanostructures, University of Goettingen, Friedrich-Hund-Platz 1, 37077 Goettingen, Germany. ${ }^{5}$ Institute for Theoretical Physics, University of Goettingen, Friedrich-Hund-Platz 1, 37077 Goettingen, Germany. ${ }^{6}$ Institute for Theoretical Physics, Clausthal University of Technology, Leibnizstr. 10., D-38678 Clausthal-Zellerfeld, Germany. ${ }^{7}$ Present address: Helmholtz-Zentrum Potsdam Deutsches GeoForschungsZentrum GFZ, Telegrafenberg 14473, Potsdam, Germany. ${ }^{8}$ Present address: HelmholtzZentrum Berlin für Materialien und Energie GmbH, Young Investigator Group Operando Interface-Photochemistry, 12489 Berlin, Germany.

$凶_{\text {email: cjooss@gwdg.de }}$
} 
T he efficiency of electrochemical water splitting for the sustainable production of clean fuels from renewable energy sources is limited by a large overpotential of the anodic oxygen evolution reaction (OER) $)^{1-3}$. Understanding of its origin requires knowledge about the pathway of the formation of di-oxygen $\mathrm{O}_{2}$ out of $2 \mathrm{H}_{2} \mathrm{O}$ molecules by a proton-coupled electron transfer. Even for the best electrocatalysts, i.e., transition metal $(\mathrm{M})$ oxides $(\mathrm{O})$, the reaction path for $\mathrm{O}-\mathrm{O}$ formation is not well understood. For heterogeneous systems, the reaction is catalyzed at active surface sites, where different sites and mechanisms are suggested. This includes single ${ }^{4}$ or dual ${ }^{5-7}$ transition metal (M) sites with flexible valence states, concerted proton-electron transfer ${ }^{8,9}$ as well as redox active lattice $\mathrm{O}^{10-12 \text {, }}$ including oxygen vacancy formation and annihilation ${ }^{13}$. Despite remarkable progress in application of density functional theory (DFT) to the oxide-water interface, the theoretical understanding of the dynamic interface structure during OER is still limited since it requires serious approximations ${ }^{14}$. Among of the farreaching are the frozen surface approximation and that solvation effects are, if at all, only considered as a static average. This underestimates the flexibility of $\mathrm{M}$ surface coordination due to interface solvent effects that is on the other hand known to be crucial in homogeneous catalysis for the formation of high- or hetero-valent $\mathrm{M}-\mathrm{O}$ moieties that precede $\mathrm{O}-\mathrm{O}$ bond formation ${ }^{15}$. There are controversial conclusions whether the predicted unsuitable scaling relation between metal $\mathrm{M}-\mathrm{OH}$ and $\mathrm{M}-\mathrm{O}$ adsorption energies ${ }^{9,16,17}$ as the origin of large overpotentials for single surface sites can be broken by bifunctional mechanism at two distinct $M$ and $M^{3}$ sites ${ }^{18}$ or is restored by surface solvation ${ }^{19}$. Recent experiments show that ternary alloying of two different transition metals with a highly flexible coordination metals indeed lead to a remarkable reduction of OER overpotential in gelled oxyhydroxides ${ }^{20}$. An improved theoretical understanding thus requires a comprehensive atomic scale understanding of the dynamic electrolyte-catalyst interface structure under OER conditions as an essential ingredient for the knowledge driven further improvement of electrocatalysts by material design.

In addition to OER activity, the stability is strongly affected by the interfacial electrolyte interactions. A distinction between reversible dynamics that emerges in a stationary state and irreversible processes that change the activity is essential. Such insights are promoted by the progress of operando studies that can address different types of dynamical changes. A first type of rather slow dynamics is the change of electrode surface during potential cycling. The important role of solvate interactions has been e.g., established for the noble metal Pt, where Pt surface oxide that is formed under anodic polarization adsorbs water that dissociates and forms hydrated $\mathrm{Pt}^{21}$. Metal oxides such as $\mathrm{CoO}_{\mathrm{x}}$ can form a disordered surface during $\mathrm{OER}^{22,23}$. A second type of rather fast dynamics is due to thermally activated mobility of surface atoms ${ }^{24}$ or adatoms $s^{25}$ that is hardly accessible in operando experiments. Clearly, surface adatom dynamics depends on temperature and surface barriers that can be modified due to $\mathrm{H}_{2} \mathrm{O}$ solvation or dissociation ${ }^{26}$ and is always present, even in thermal equilibrium. Driving the OER, the emerging non-equilibrium surface should show a reversible dynamics in a stationary state that depends on the formed atomic and chemical surface structure. Non-equilibrium dynamics of catalyst atoms may be even involved in OER mechanism ${ }^{27}$. A third type of surface dynamics is irreversible defect reactions, typically the formation of oxygen vacancies $^{13,28}$ or the dissolution of metallic species ${ }^{29}$. Such processes are even gradually present at the highest performance system $\mathrm{RuO}_{2}{ }^{30}$. Metal dissolution can depend on overpotential ${ }^{31,32}$ surface symmetry ${ }^{33}, \mathrm{pH}$ value and particle size $^{34}$. In order to avoid such corrosive processes, approaches for self-repair have been pursued ${ }^{35}$. Despite a huge progress in application of different operando techniques ${ }^{36}$, comprehensive atomic scale understanding of all three classes of surface dynamics are far of reach at present, but are urgently required to establish the intimate connections between surface stability, the nature of the active sites and reaction pathways ${ }^{37}$.

In situ environmental transmission electron microscopy (ETEM) in combination with aberration correction and image simulation has developed to a level where it can provide atomic resolution real space information about the equilibrium surface reconstruction in various ambient conditions ${ }^{38-40}$ including strategies for controlling the impact of electron beam ${ }^{41,42}$. The recent development of atomic scale dynamic studies show surface mobility of Ce atoms on $\mathrm{CeO}_{2}$ facets in high vacuum ${ }^{43}$ and a transition of a $\mathrm{TiO}_{2}$ surface reconstruction in $\mathrm{H}_{2} \mathrm{O}$ from static to dynamic after adding $\mathrm{CO}^{44}$. Steps towards the control of electron beam-induced and applied electric potentials at TEM samples enable in situ studies of electrode surfaces in ETEM under anodic polarization, approaching OER relevant potentials ${ }^{28,45}$.

In this study, two perovskite manganites $\mathrm{AMnO}_{3}$ were selected, where the Mn valence state can be controlled by A-site doping without changing the crystal symmetry. Specifically, $\mathrm{La}_{1-\mathrm{x}} \mathrm{Sr}_{\mathrm{x}} \mathrm{MnO}_{3}$ at $\mathrm{x}=0.4$ (LSMO) is a metal-like electrode with high and stable oxygen evolution activity that is close to $\mathrm{IrO}_{2}{ }^{29}$. Moreover, $\operatorname{Pr}_{1-\mathrm{x}} \mathrm{Ca}_{\mathrm{x}} \mathrm{MnO}_{3}$ at $\mathrm{x}=0.33$ (PCMO) is a semiconducting small polaron material with high initial OER activity, yet less stability, due to oxygen vacancy formation and fast leaching of the resulting reduced Mn species. Thus LSMO is suitable for the study of reversible surface dynamics, whereas PCMO shows a pronounced irreversible defect reaction. Both systems have almost the same initial Mn valence but differ in $\mathrm{Mn}-\mathrm{O}$ covalency and charge localization. This differences allows us to correlate in situ ETEM studies of surface structure and dynamics with ex situ analysis of electrochemical OER. We find that the (001) surfaces of both manganites show an at least 20 times higher Mn-adatom mobility in an adsorbed water layer compared to other ambients.

\section{Results and discussion}

Structure, activity, and stability of LSMO and PCMO (001). Rotating ring disk electrode (RRDE) cyclovoltammetry (CV) was carried out at epitaxial (001) oriented thin film electrodes in $0.1 \mathrm{M}$ aqueous $\mathrm{KOH}$ solution (Fig. 1). Both materials initially show good electrocatalytic oxygen evolution performance (for oxygen detection and Tafel analysis see Supplementary Figs. 1-3). Most striking is the different behavior just below the onset of the OER, where a redox wave is visible in PCMO as a negative current peak at $E=1.3 \mathrm{~V}$ vers reversible hydrogen electrode (RHE), complemented by a positive shoulder at the onset of OER. This feature is absent in LSMO. The redox couple is related to reversible formation and annihilation of oxygen vacancies, which are electron donors and thus induce a surface reduction of the transition metal ${ }^{13}$. The defect reaction is given by

$$
\mathrm{Pr}_{1-\mathrm{x}} \mathrm{Ca}_{\mathrm{x}} \mathrm{Mn}^{2+\mathrm{x}} \mathrm{O}_{2.5}+\mathrm{V}_{\mathrm{o}}^{*}+1 / 2 \mathrm{O}_{2} \geq \mathrm{Pr}_{1-\mathrm{x}} \mathrm{Ca}_{\mathrm{x}} \mathrm{Mn}^{3+\mathrm{x}} \mathrm{O}_{3}+2 \mathrm{e}^{-}
$$

For neutral $\mathrm{pH}$ values in an unbuffered solution, which is more relevant for the comparison to in situ XANES and ETEM studies, a similar behavior of the $\mathrm{CV}$ is observed ${ }^{13}$. The easy formation of $\mathrm{V}_{\mathrm{o}}^{*}$ in PCMO indicates that lattice oxygen is redox active, such that transfer of holes between Mn3d and O2p states can oxidize $\mathrm{O}^{2-}$ to form $\mathrm{O}^{-}$and $\mathrm{O}_{2}(\mathrm{~g})$; see e.g., refs. ${ }^{46,47}$. For LSMO such a surface redox process is only observed when the cycling is extended to more negative potentials below $E=0.6 \mathrm{~V}$ vers $\mathrm{RHE}^{48}$.

Comparing the cycle dependence of the OER activity for LSMO and PCMO in Fig. 1b, there is a strong difference in 

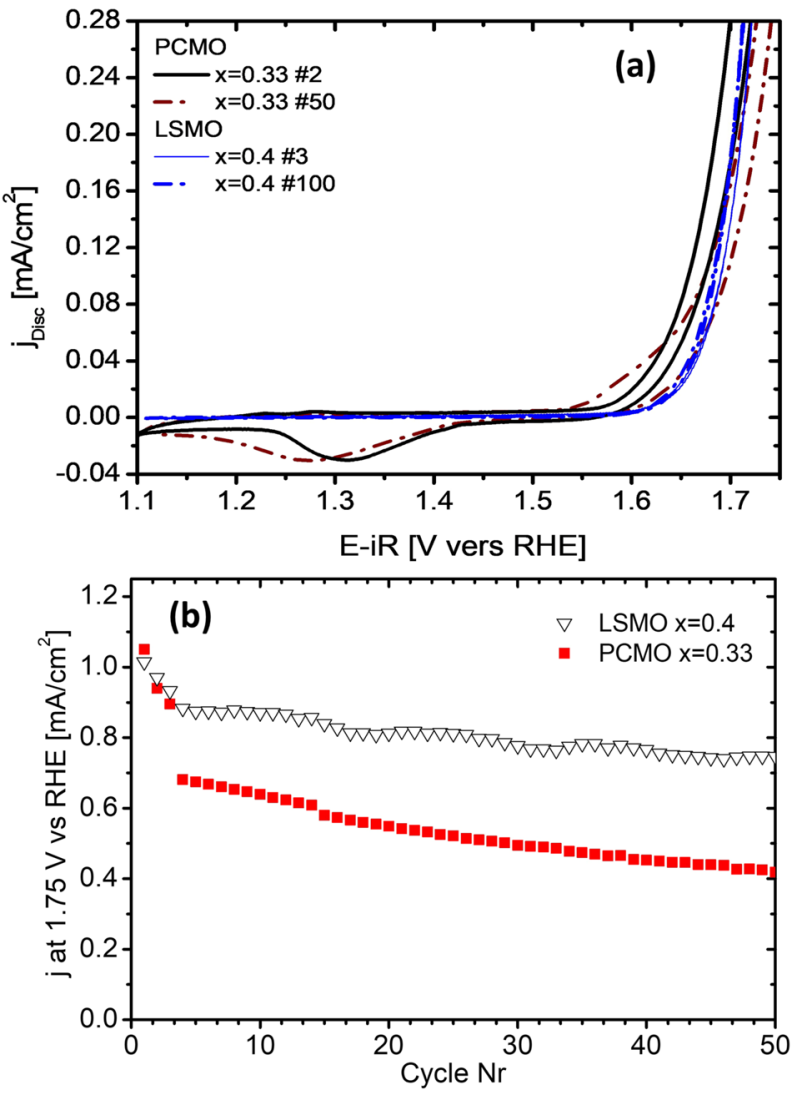

Fig. 1 Electrocatalytic characterization. a Cyclovoltammetry of (001) oriented epitaxial thin film manganite electrodes in $0.1 \mathrm{M}$ aqueous $\mathrm{KOH}$ solution at $10 \mathrm{mV} / \mathrm{s}$ and rotated at $1600 \mathrm{rpm}$ for cycles \#2/\#50 (PCMO) and \#3/\#100 (LSMO), respectively. In addition to the OER, PCMO shows a pronounced surface redox couple. $\mathbf{b}$ Temporal stability of the electrode current density at $E=1.75 \mathrm{~V}$ vers. RHE for cycling from $E=1.1-1.75 \mathrm{~V}$ vers. RHE and 7 min chronoamperometric OER between cycle 3 and 4 (see method section).

stability between both electrodes. Initially, the current density $j$ of both systems at $E=1.75 \mathrm{~V}$ vers. RHE is similar, i.e., $j=$ $1.03 \mathrm{~mA} / \mathrm{cm}^{2}$ for LSMO and $j=1.05 \mathrm{~mA} / \mathrm{cm}^{2}$ for PCMO. These values are close to the activity of $\mathrm{IrO}_{2}$ (110) films ${ }^{49}$. However, upon cycling and chronoamperometric OER between cycle 3 and 4, the decrease for PCMO is much more pronounced than for LSMO. Comparing the pristine and post electrolysis structure of LSMO and PCMO after 50 electrochemical cycles (Supplementary Figs. 4-7) reveals significant differences. Whereas for LSMO the crystalline surface structure with flat terraces and unit cell height steps is fully preserved, PCMO displays an increase of surface roughness. Postmortem high-resolution scanning transmission electron microscopy (HRSTEM) combined with electron energy loss spectroscopy (EELS) of film surfaces shows a strong surface depletion of Mn for PCMO, leading to a 2-3 nm thick Prenriched surface. Emerging small peaks in XRD after $1 \mathrm{~h}$ of electrolysis are consistent with a thin cubic $\mathrm{PrO}_{2+x}$ layer. In contrast, the Mn concentration at the LSMO surface is only slightly reduced.

Surface termination of the manganite films in $\mathrm{H}_{2} \mathrm{O}$. The study of surface termination of epitaxial perovskite films using environmental high-resolution transmission electron microscopy (ETEM) was demonstrated by Roddatis et al. ${ }^{42}$ and requires careful TEM lamella preparation, as documented in the Supplementary Figs. 8-11 and the associated text. The changes of surface termination of the LSMO and PCMO (001) oriented thin film electrodes in high vacuum (HV), $\mathrm{O}_{2}$ and $\mathrm{H}_{2} \mathrm{O}$ are shown in Fig. 2. For LSMO predominant A-terminated surfaces ( $\mathrm{La}-\mathrm{Sr}-\mathrm{O}$ ) are observed in $\mathrm{O}_{2}$, whereas in high vacuum $(\mathrm{HV})$, a mixed surface termination with disorder at the B-terminated ( $\mathrm{Mn}-\mathrm{O})$ areas is developed. This is observed even after saturation of the surface in 1 mbar $\mathrm{O}_{2}$. In $\mathrm{H}_{2} \mathrm{O}$ vapor, the LSMO surface appears to be dominated by an A-site layer; however, $\mathrm{Mn}$ is still present as dynamic surface adatoms. For PCMO, a disordered surface develops in $\mathrm{HV}$ following the ordering of the surface in $1 \mathrm{mbar}$ of $\mathrm{O}_{2}$, where a mixed termination of an ordered surface is observed. In $\mathrm{H}_{2} \mathrm{O}$, a mixed surface termination emerges, where the Aterminated areas ( $\mathrm{Pr}-\mathrm{Ca}-\mathrm{O})$ show higher ordering compared to the B-terminated areas. Surface $\mathrm{Mn}$ adatoms as well as subsurface lattice Mn is highly mobile.

The determination of surface termination requires the comparison of the HRTEM images with simulated images (Fig. 2d, h). In these simulations, the lamella thickness $t$ at the surface is estimated from measurement of the inelastic scattering intensity by EELS and fine-tuned by a fit of contrast of simulated to experimental images using a Monte-Carlo-based simulated least-squares optimization, yielding $t=4.2(4.3) \mathrm{nm}$ for LSMO (PCMO). The obtained electron optical parameters, such as defocus, spherical aberration, astigmatism, and axial coma, fit reasonably well to those estimated from tilt-tableau measurements via the Cs-corrector software. The different surface terminations can be clearly distinguished by the weaker contrast of the $\mathrm{MnO}$ columns. In the subsurface, A-site and B-site columns are separable from contrast due to different atomic numbers and octahedral tilt patterns of $\mathrm{MnO}_{6}$ octahedra. Details of the image fitting and parameters used can be found in the Supplementary Fig. 12 and Supplementary Table 1.

Reversible Mn-adatom dynamics at the LSMO- $\mathrm{H}_{2} \mathrm{O}$ interface. Figure 3 shows a time sequence of HRTEM images for the LSMO $\mathrm{x}=0.4$ (001) surface in $0.5 \mathrm{~Pa}$ of $\mathrm{H}_{2} \mathrm{O}$. The full image series is compiled in Movie M01. The surface is well ordered and shows stable A-termination over more than $30 \mathrm{~min}$. However, mobile $\mathrm{Mn}$ is, in fact, present on the surface. This is indicated by the sudden appearance and disappearance of atom contrast at B-layer surface positions on flat stable A layers (Fig. 3a). We define the detection limit of $\mathrm{Mn}$ atoms by the amplitude of the signal above the noise level $3 \sigma$, where $\sigma$ is the standard deviation of the background fluctuations of the CCD signal at an area above the surface in the presence of the experimental gas. Image simulation and contrast quantification show that signal to noise ratio at the used frame rate of $4.4 \mathrm{fps}$ does allow for detection of triple or higher occupancy of B-columns by $\mathrm{Mn}$ at a $4.2-\mathrm{nm}$ thick lamella surface (Fig. 3b, c).

Closer inspection of image contrast in Fig. 3a reveal adatom contrast mainly appears at interstitial surface positions and rarely at the nominal octahedral positions of an ideal non-reconstructed surface. Contrast quantification of the columns of $\mathrm{Mn}$ adatoms is obtained by calibration of the experimental A-site contrast at the lamella surface to the simulated contrast, as shown in the Supplementary Figs. 13-15. Based on contrast simulations, the interpretation of dynamic adatom contrast other than $\mathrm{Mn}$ on a static A-layer is unlikely: $\mathrm{O}$ and $\mathrm{OH}$ species are below the contrast detection limit. La/Sr occupancy on B-terminated layers can be excluded from contrast. Fast $\mathrm{La} / \mathrm{Sr}$ adatom dynamics on a stable A-terminated layer can be not entirely excluded from contrast. However, this would lead to an A-enriched surface with a Mn depleted layer, which is contrary to our observations for LSMO but in agreement to $\mathrm{Mn}$ surface leaching in PCMO reported below. Since all experiments in different environments 


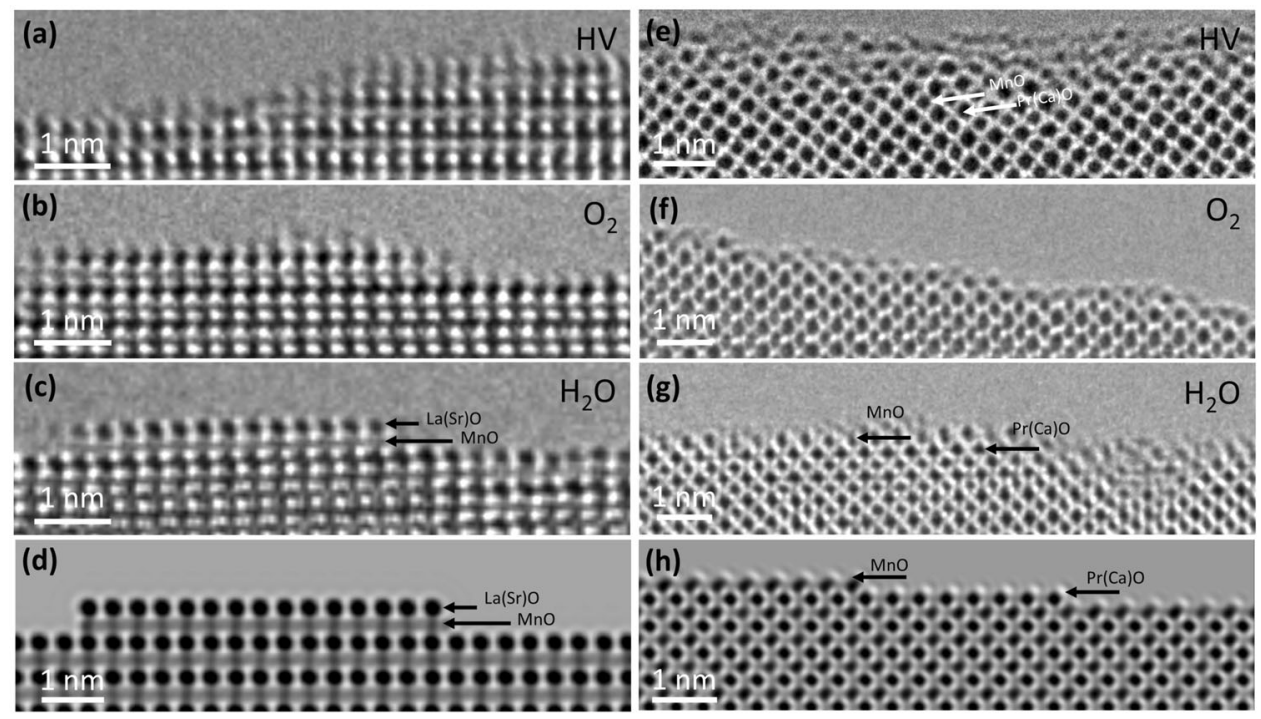

Fig. 2 HRTEM cross plane images of LSMO and PCMO (001) surfaces in different ambients. All images are taken at negative defocus, i.e., atomic columns appear as dark contrast, and with objective lens aberrations corrected up to 5th order. a-d LSMO is imaged in the pseudocubic [110] zone axis. a Mixed B- and A-termination in high vacuum (HV). b Dominant A-termination in 1 mbar $\mathrm{O}_{2}$. c In $0.5 \mathrm{~Pa} \mathrm{H}_{2} \mathrm{O}$, the surface consists of a stable A-layer with highly mobile $\mathrm{Mn}$ on top (see Fig. 3). d Simulated image with A-termination and surface step. $\mathbf{e}-\mathbf{h}$ PCMO is imaged in the orthorhombic [110] zone axis. e Disordered surface structure in $\mathrm{HV}$. $\mathbf{f}$ Highly ordered surface in 1 mbar of $\mathrm{O}_{2}$ with a mixed termination. $\mathbf{g} \ln 0.5 \mathrm{~Pa}$ of $\mathrm{H}_{2} \mathrm{O}$, the B-terminated areas of PCMO exhibit pronounced disorder with mobile Mn. h Simulated image for PCMO with different surface terminations.

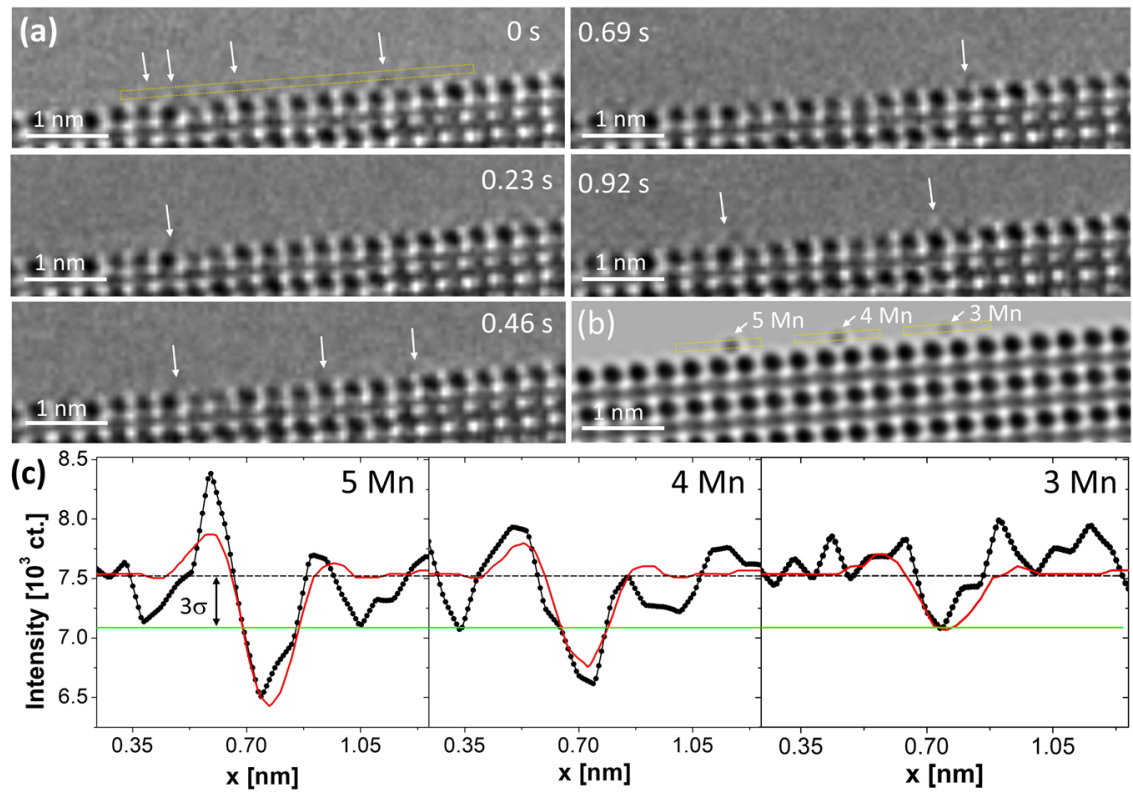

Fig. 3 Observation of dynamic Mn adatoms on a LSMO (001) surface in $\mathbf{0 . 5} \mathbf{P a}$ of $\mathbf{H}_{\mathbf{2}} \mathbf{O}$ at [110] zone axis. a Time sequence of HRTEM images taken at $4.4 \mathrm{fps}$ at negative defocus. Mn-adatom contrast appears and disappears on a stable La(Sr)O surface layer as indicated by white arrows. b Simulated HRTEM image with Mn-adatom column occupation of 3, 4, and 5. The Mn columns are located at interstitial surface positions on an A-terminated sample. Lamella thickness is $4.2 \mathrm{~nm}$. c Line profiles of simulated (red) and experimental (black with symbols) images of B-columns with 3, 4, and 5 Mn atom occupation. The $3 \sigma$ threshold indicates the detection limit of Mn columns above noise level, i.e., triple Mn occupation at a B-layer column. The time sequence gives a lower limit of the Mn-adatom hopping rate of $r \geq 4 \mathrm{~s}^{-1}$.

reveal that surface $\mathrm{Mn}$ is more mobile than A-type cations, our overall conclusion from image contrast and electrochemical behavior is that $\mathrm{Mn}$ adatoms are the highly mobile species on stable LSMO surfaces in $\mathrm{H}_{2} \mathrm{O}$.

The camera frame rate of around $4 \mathrm{fps}$ is selected as a compromise between achieving sufficient signal to noise ratio and capturing some of the surface dynamics. Double Mn B-layer occupancy corresponds to the detection limit at $3 \sigma$ of the noise level. Both triple $\mathrm{Mn}$ as well as higher Mn B-layer occupancy contrast appears and disappears with the frame rate used. We can thus only determine a lower limit of the surface hopping rate, which is given by the frame rate of the images. If triple $\mathrm{Mn}$ occupation appears and disappears on the timescale of the frame rate, it may be attributed to one of the following events: (i) A single $\mathrm{Mn}$ atom hops to another site and the remaining double occupation contrast becomes invisible. (ii) Two or three Mn atoms coincidentally hop to another site and the B-site contrast on the original site disappears. Since (ii) has a lower probability 

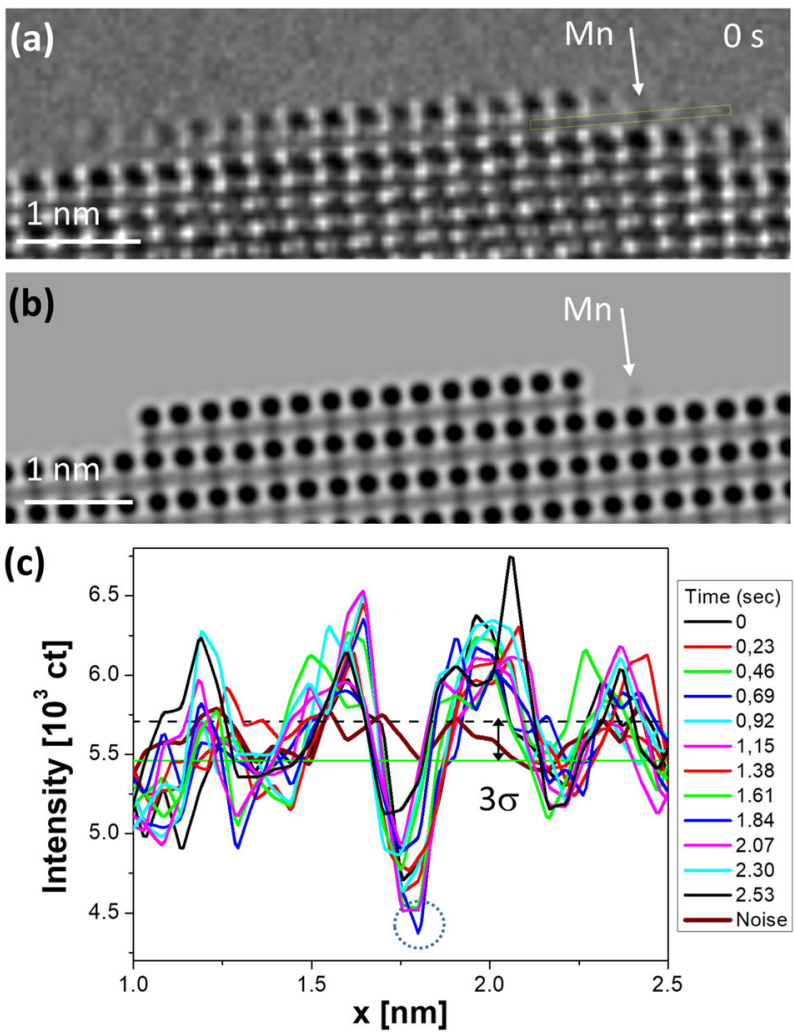

Fig. 4 Slowed down Mn surface mobility due to a unit cell height surface step at a LSMO (001) facet in $\mathbf{0 . 5} \mathbf{P a}$ of $\mathbf{H}_{\mathbf{2}} \mathbf{O}$. a First HRTEM image of a time sequence taken at a frame rate of $4.4 \mathrm{fps}$ with the surface step taken at the same conditions as in Fig. 3; see Movie M02. b Simulated HRTEM image with the step and $3 \mathrm{Mn}$ occupation contrast on a slightly displaced Bsite column. $\mathbf{c}$ Line profiles of the time sequence taken at the B-layer as indicated by the green box in $\mathbf{a}$. It is showing an increased sticking time of a triple Mn column of $1.4 \mathrm{~s}$ near the step edge compared to $\leq 0.23 \mathrm{~s}$ at the flat surface, providing evidence for a locally increased surface barrier.

than (i) and represents the slowest process, it defines the minimum detectable hopping rate of $r \geq 4 \mathrm{~s}^{-1}$.

The dynamic state with highly mobile Mn atoms on top of a more or less static A-terminated layer can be strongly affected by the presence of a surface step. Since the Mn surface mobility depends on the surface activation barrier, it can be strongly reduced at step edges due to an Erich-Schwöbel barrier. Figure 4 shows an increased contrast of mobile $\mathrm{Mn}$ atoms in the vicinity of step edges formed by a static A-terminated layer. The reduction of Mn mobility in the vicinity of the step edges can directly be observed in the Movie M02. The analysis of line profiles at the Blayer location in the vicinity of the step edge in Fig. 4 reveal a reduction of the Mn-adatom hopping rate from $r \geq 4 \mathrm{~s}^{-1}$ to $r \approx$ $0.7 \mathrm{~s}^{-1}$. Remarkably, the A-layer forming the step edge in Fig. 4 is quite stable and does not change on the timescale of the Movie M02 of 1 min 25; see also Supplementary Fig. 16.

Compared to $\mathrm{H}_{2} \mathrm{O}$, the Mn surface mobility on LSMO (001) in high vacuum (HV) and in other gases is strongly reduced at the same dose rate (Supplementary Figs. 17-19, Movies M03-M05). The surface with mixed termination shows contrast changes on the B-site columns with $r \leq 0.25 \mathrm{~s}^{-1}$ in $\mathrm{HV}$ and $r \leq 0.2 \mathrm{~s}^{-1}$ in 100 $\mathrm{Pa} \mathrm{O}_{2}$, where the highly ordered surface is predominantly A-site terminated. The high Mn surface mobility on LSMO (001) is thus a unique observation in $\mathrm{H}_{2} \mathrm{O}$ and is visible in a pressure range of 0.01-5 Pa. At higher $\mathrm{H}_{2} \mathrm{O}$ pressures, LSMO becomes unstable, possibly due to the impact of ions formed by beam-induced ionization of $\mathrm{H}_{2} \mathrm{O}$. Despite the high mobility, the $\mathrm{Mn}$ leaching is rather slow, as confirmed by the post-ETEM stoichiometry analysis (Supplementary Fig. 20). Furthermore, the Mn valence state remains quite stable, indicating only a slight $\mathrm{Mn}$ reduction in increasing $\mathrm{H}_{2} \mathrm{O}$ pressure and ruling out pronounced formation of oxygen vacancies.

Irreversible Mn-adatom dynamics at the PCMO- $\mathrm{H}_{2} \mathrm{O}$ interface. Figure 5 shows a time series of HRTEM images of the (001) PCMO surface in $0.5 \mathrm{~Pa} \mathrm{H}_{2} \mathrm{O}$, taken from Movie M06. After fully ordering in $\mathrm{O}_{2}$ (Fig. 2f), the addition of $\mathrm{H}_{2} \mathrm{O}$ causes the surface to form a disordered layer of highly mobile $\mathrm{Mn}$ atoms, visible as rapid fluctuation of B-site contrast. Similar to LSMO, the hopping rate of $\mathrm{Mn}$ atoms is above the frame rate of the camera, i.e., $r \leq 4 \mathrm{~s}^{-1}$. In contrast to the disordered B-terminated surface, the A-subsurface layer remains highly ordered and static on the timescale of several seconds (Movie M06). In addition, an increase of contrast dynamics is observed at the B-site columns at the subsurface. Figure $5 b, c$ shows the later stages of the PCMO (001) surface in $0.5 \mathrm{~Pa} \mathrm{H}_{2} \mathrm{O}$. Three min after initiation, the fast Mn leaching has led to the formation of a Pr-rich surface bilayer (Movie M06) which further grows up to a thickness of 3-4 monolayers over the course of the in situ experiment (11 min). The growth of a cubic phase due to Mn leaching is directly visible in the surface structure. Postmortem EELS analysis of the surface area in Fig. $5 \mathrm{f}$ reveals $\mathrm{Pr}$ and $\mathrm{O}$, whereas the Mn L-edge at $640 \mathrm{eV}$ as well as Ca L-edge at $346 \mathrm{eV}$ (not shown) are below the noise level. We thus associate the cubic oxide visible in HRTEM with $\mathrm{PrO}_{\mathrm{x}}$, with $\mathrm{x}$ close to 2 . The growth speed of the $\mathrm{PrO}_{\mathrm{x}}$ slows down over time, indicating self-passivation of the surface by the formed $\mathrm{PrO}_{\mathrm{x}}$ layer. Post-ETEM EELS of the $\mathrm{Mn} \mathrm{L}$ and $\mathrm{O} \mathrm{K}$ edges at the PCMO surface region reveals a pronounced Mn depletion (Supplementary Fig. 21), which is accompanied by $\mathrm{Mn}$ reduction. Leaching out of subsurface Mn in PCMO (001) is only observed in $\mathrm{H}_{2} \mathrm{O}$ and is absent in $\mathrm{HV}$ (Movie M07) and $\mathrm{O}_{2}$ (Movie M08). In contrast, the $\mathrm{Mn}$ concentration at LSMO surfaces remains unmodified. This is fully consistent with the observation of different electrode behavior in electrochemical experiments described above.

Enhanced Mn mobility at (001) surface due to presence of adsorbed $\mathrm{H}_{2} \mathrm{O}$ layer. The formation of a few monolayer thick water layer on oxide surfaces down to pressures of $\sim 10^{-5} \mathrm{~Pa}$ much below the equilibrium vapor pressure of water of $\mathrm{p}_{\mathrm{vap}}=3.17 \mathrm{kPa}$ at $\mathrm{T}=25^{\circ} \mathrm{C}$ has been previously demonstrated ${ }^{50-52}$. It is driven by the large adsorption enthalpy of $\mathrm{H}_{2} \mathrm{O}$ on oxide surfaces of the order of $\Delta H_{a d, o x}-160 \mathrm{~kJ} / \mathrm{mol}^{51,53}$. Although this value might vary between different oxides, $\Delta H_{a d, o x}<\Delta H_{a d}=-44 \mathrm{~kJ} / \mathrm{mol}$ for $\mathrm{H}_{2} \mathrm{O}$ on bulk water reflects exothermic water adsorption on oxide surface at much reduced pressures. In the HV of the ETEM $(p \geq$ $10^{-5} \mathrm{~Pa}$ ), the effect of $\mathrm{H}_{2} \mathrm{O}$ condensation on manganite surfaces can be only avoided by using a cold trap that is reducing the partial pressure of $\mathrm{H}_{2} \mathrm{O}$ in $\mathrm{HV}$ by one order of magnitude ${ }^{42}$. The thickness of the adsorbed water layer in the ETEM experiments as a function of $\mathrm{p}_{\mathrm{H}_{2} \mathrm{O}}$ is calculated by using the energy balance between surface energy $\gamma_{\mathrm{s}}$ of the oxide in $\mathrm{HV}$, the surface energy $\gamma_{\mathrm{H}_{2} \mathrm{O}}$ of liquid $\mathrm{H}_{2} \mathrm{O}$ to vapor and $\Delta H_{a d}$ (see Supplementary Fig. 22 and associated text). The liquid state of $2-4$ monolayer $\mathrm{H}_{2} \mathrm{O}$ is consistent with our observation of enhanced mobility of $\mathrm{Mn}$ on manganite (001) surfaces in a pressure range between 0.01 and $5 \mathrm{~Pa}$ of $\mathrm{H}_{2} \mathrm{O}$ compared to $\mathrm{HV}$.

Thermally induced versus electron beam-induced hopping. In order to separate out the effect of the electron beam on adatom motion from the thermally induced motion, the momentum 

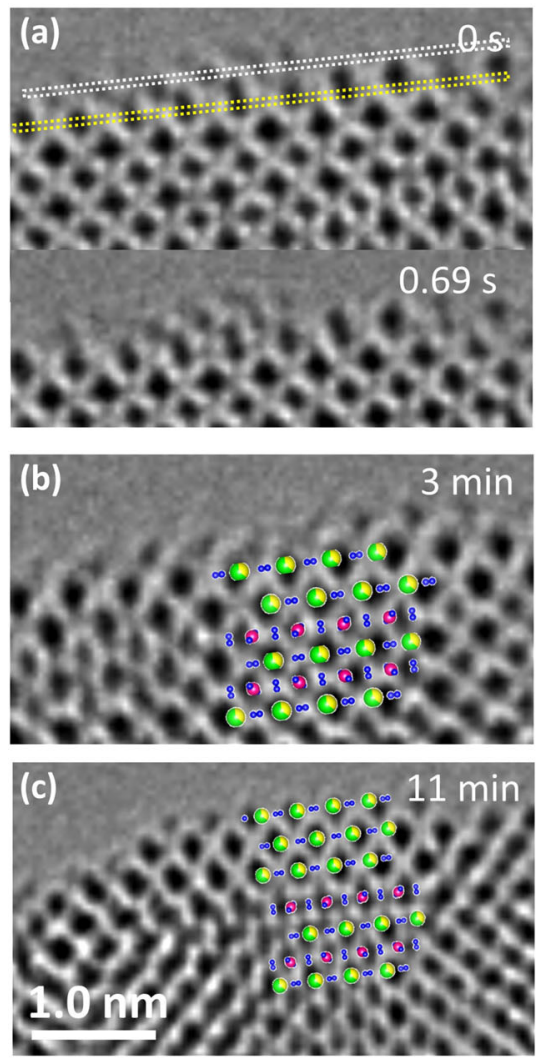
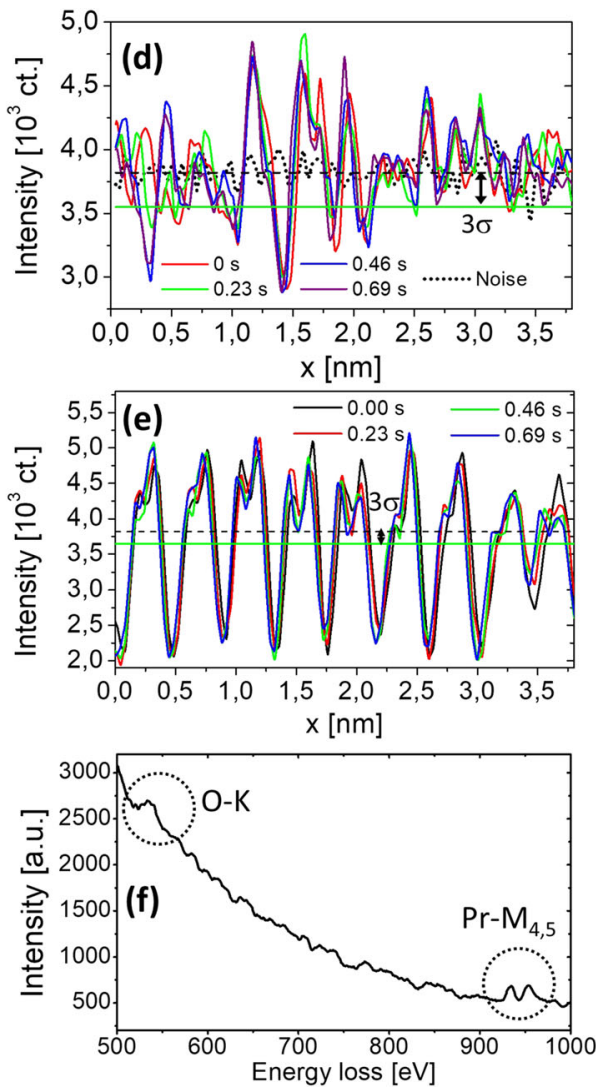

Fig. 5 Atomic surface dynamics of the PCMO (001) surface in $\mathbf{0 . 5} \mathbf{P a} \mathbf{H}_{\mathbf{2}} \mathbf{O}$. a HRTEM images taken from a time series taken at a rate of 4 fps at [110] zone axis and negative defocus, compiled in Movie M06. b, c Later stages of that surface area at same conditions. The line profiles are taken at the B-site (d) and A-site (e) columns. Whereas the A-site columns of the first subsurface layer remain ordered (f), the B-site columns transform into a dynamical highly disordered surface structure. The measured hopping rate of $\mathrm{Mn}$ adatoms is $r \geq 4 \mathrm{~s}^{-1}$, from triple Mn coincidence occupation. In addition to surface movement, there is a pronounced leaching of $\mathrm{Mn}$ from the subsurface to the surface leading to the growth of a Pr-rich surface layer, where $\mathrm{Mn} \mathrm{L}_{3,2}$ is below the detection limit $(\mathbf{f})$.

transfer from scattering of the high energy primary electron beam on the adatoms is calculated. As pointed out by Egerton $^{54}$, for energies of primary electrons $E_{0} \geq 10 \mathrm{keV}$, the interaction is dominated by elastic scattering of the incident electrons at the atomic nuclei, and momentum transfer due to inelastic scattering at the electron shell can be disregarded. This allows calculation of the momentum transfer using the Rutherford scattering equation. We consider the maximum momentum transfer perpendicular to the primary electron beam, which gives rise to transversal movement of adatoms on a surface parallel to the electron beam. Figure 6 compares the beam-induced hopping rates for $\mathrm{O}, \mathrm{Mn}, \mathrm{Sr}$ and $\mathrm{La}$ adatoms to the thermally activated hopping rate as a function of the surface activation energy $E_{a}$ for an upper limit of used dose rates of $12.000 \mathrm{e}^{-} / \AA^{2} \mathrm{~s}$ (see method section). For $E_{a} \geq$ $0.2 \mathrm{eV}$, the calculated beam-induced hopping rate for Mn adatoms is below the experimentally observed hopping rate in HV, indicating that the slow $\mathrm{Mn}$ motion is thermally activated. The comparison of the calculated thermally activated hopping with the experimentally observed $r(\mathrm{HV}) \approx 0.25 \mathrm{~s}^{-1}$ leads to an estimated $E_{a}(\mathrm{Mn}) \approx 0.75 \mathrm{eV}$ in $\mathrm{HV}$. The increase of the $\mathrm{Mn}$-adatom hopping in $\mathrm{H}_{2} \mathrm{O}$ to $r\left(\mathrm{H}_{2} \mathrm{O}\right) \geq 4 \mathrm{~s}^{-1}$ thus can be interpreted as resulting from a reduction of the effective surface activation barrier in the presence of a $\mathrm{H}_{2} \mathrm{O}$ layer to $E_{a}(\mathrm{Mn})<0.62 \mathrm{eV}$ most probably due to partial solvation of surface $\mathrm{Mn}$ in $\mathrm{H}_{2} \mathrm{O}$. Furthermore, the calculation of momentum transfer between the electron beam and $\mathrm{H}_{2} \mathrm{O}$ shows that the adsorbed $\mathrm{H}_{2} \mathrm{O}$ layer is only marginally affected by the beam since the beam-induced desorption rate is with $\approx 2.510^{-3} \mathrm{~s}^{-1}$ orders of magnitudes below the impingement rate of $\mathrm{H}_{2} \mathrm{O}$ molecules of around $10^{3} \mathrm{~s}^{-1}$ at $p=$ $0.5 \mathrm{~Pa}$.

Parallel trends in electrolysis and ETEM studies with respect to reversible and irreversible dynamics. For LSMO $\mathrm{x}=0.4$, the (001) interface to liquid $\mathrm{H}_{2} \mathrm{O}$ is quite stable both in in situ ETEM experiments as well as in cyclovoltammetry in alkaline conditions between +1.1 and $1.75 \mathrm{~V}$ vers. RHE. Only slow $\mathrm{Mn}$ leaching is observed in previous studies by postmortem XPS after $1 \mathrm{~h}$ of electrochemical OER, which can be further slowed down by choosing a $\mathrm{Mn} / \mathrm{A}$ ratio $\geq 1$ ref. ${ }^{55}$. In contrast to LSMO, the in situ ETEM study of PCMO shows pronounced irreversible surface dynamics of $\mathrm{Mn}$, leaching into the liquid $\mathrm{H}_{2} \mathrm{O}$ layer in parallel to fast drop of OER activity in electrolysis and observation of a $\mathrm{Pr}$ rich surface layer in postmortem studies. The huge difference in reversibility of $\mathrm{Mn}$-adatom motion at the interface between the more or less static A-site terminated layer and liquid $\mathrm{H}_{2} \mathrm{O}$ must therefore be attributed to the different materials properties of LSMO and PCMO. Post-ETEM electron energy loss spectroscopy (EELS) of the $\mathrm{Mn} \mathrm{L}$ edge at $\mathrm{a} \approx 1$-nm thick surface layer on PCMO demonstrates a change of Mn valence from $3.5 \pm 0.1$ after oxidation in $\mathrm{O}_{2}$ to $3.2 \pm 0.1$ after ETEM study in $0.5 \mathrm{~Pa}$ and $3.1 \pm$ 0.1 in $5 \mathrm{~Pa}$ of $\mathrm{H}_{2} \mathrm{O}$ (Supplementary Fig. 21). Thus, $\mathrm{Mn}$ is reduced although the experiments are performed under anodic polarization. In contrast, for LSMO the pristine Mn valence of $3.5 \pm 0.1$ after oxidation in $\mathrm{O}_{2}$ and $3.4 \pm 0.1$ after ETEM study in $0.5 \mathrm{~Pa}$ $\mathrm{H}_{2} \mathrm{O}$ is preserved within the error of measurements (Supplementary Fig. 20). Previous post electrochemical XPS studies of 


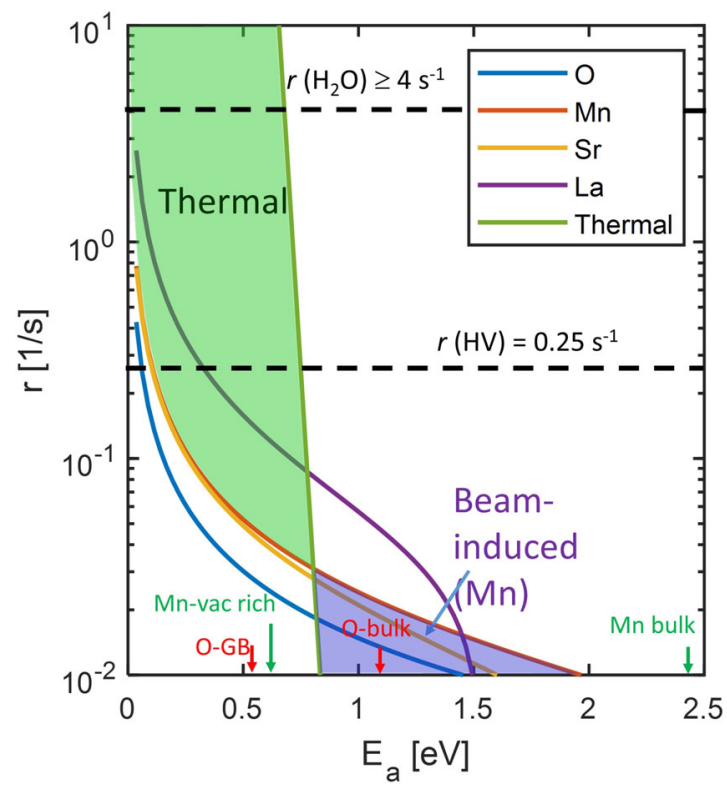

Fig. 6 Calculated beam-induced and thermally activated adatom hopping rate $r$ at $\mathbf{T}=22^{\circ} \mathrm{C}$ as a function of the surface activation energy $E_{a}$.

Additionally, the experimentally observed $\mathrm{Mn}$-adatom hopping rates in high vacuum $r(\mathrm{HV})$ and $r\left(\mathrm{H}_{2} \mathrm{O}\right)$ in $0.5 \mathrm{~Pa} \mathrm{H}_{2} \mathrm{O}$ are indicated. Colored areas mark the regimes for thermally and beam-induced hopping for $\mathrm{Mn}$. The activation energy of $\mathrm{O}$ diffusion in bulk LMO is from ref. 65 and for $\mathrm{O}$ in $\mathrm{Sr}$ doped LMO grain boundaries from ref. ${ }^{66}$. For the activation energy of $\mathrm{Mn}$ the lower and upper limit of $\mathrm{Mn}$ diffusion in LMO are indicated, since it shows a broad range of values, depending on the La-off-stochiometry of the sample ${ }^{67}$. See method section for details of the calculations.
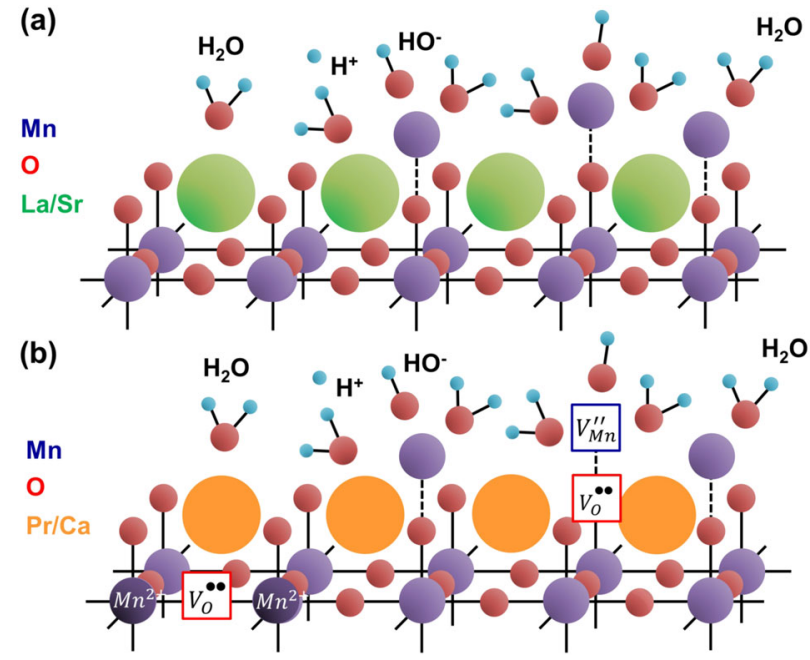

Fig. 7 Schematic representation of a snapshot of the dynamic manganite$\mathbf{H}_{\mathbf{2}} \mathbf{O}$ interface. a For LSMO, the observed reversible Mn-adatom dynamics in liquid $\mathrm{H}_{2} \mathrm{O}$ is connected to the absence of defect reactions and preservation of $\mathrm{Mn}$ oxidation state. $\mathbf{b}$ In contrast for $\mathrm{PCMO}$, the reduced $\mathrm{Mn}^{2+}$ species are formed, due to surface and subsurface oxygen vacancy $V_{\circ}^{*}$ formation.

LSMO (001) do not show surface loss of oxygen ${ }^{29}$. As summarized in Fig. 7, these observations suggest that stabilization of high oxidation states of surface $\mathrm{Mn}$ is essential for a high reversibility of $\mathrm{Mn}$-adatom movement and thus for the stability on the surface. Surface reduction and $\mathrm{Mn}^{2+}$ formation lead to irreversible Mn surface dynamics due to the higher solubility of $\mathrm{Mn}^{2+}$ in
$\mathrm{H}_{2} \mathrm{O}$ compared to $\mathrm{Mn}^{3+}$. Leaching of $\mathrm{Mn}^{2+}$ in $\mathrm{H}_{2} \mathrm{O}$ is also predicted by a Pourbaix diagram of binary $\mathrm{Mn}-\mathrm{O}$ compounds.

We attribute the different behavior of LSMO and PCMO to their different electronic properties, in particular covalency and charge localization (see Supplementary Figs. 23 and 24). Metallic LSMO exhibits spatially delocalized charge carriers, so called large polarons due to a larger $\mathrm{Mn}-\mathrm{O}$ covalency and an electronphonon coupling constant $\alpha \approx 1^{56}$. As long as the metallic state is preserved at the electrode surface, an extra charge acquired from water oxidation cannot change the valence state of a single atomic surface species. In contrast, PCMO $\mathrm{x}=0.33$ displays localized charge carriers, so called small polarons due a smaller $\mathrm{Mn}-\mathrm{O}$ covalency and larger $\alpha \approx 3$ (see ref. ${ }^{57}$ ). They represent Zener polarons, which are located on $\mathrm{Mn}-\mathrm{O}-\mathrm{Mn}$ bridges ${ }^{58-60}$. From the chemical perspective, such a Zener polaron represents the formation of an O-oxo species, due to the presence of a localized hole charge and thus the oxidation of the lattice oxygen with a formal valence state of $\mathrm{O}^{2-}$. Under anodic polarisation, hole type polarons can accumulate on the surface near $\mathrm{Mn}-\mathrm{O}-\mathrm{Mn}$ bridges and thus can easily induce oxidation of lattice oxygen to $\mathrm{O}^{-}$as an intermediate step for subsequent formation of vacancies $V_{O}$. Formation of $\mathrm{O}^{-}$and $\mathrm{V}_{\mathrm{O}}$ thus can therefore reduce $\mathrm{Mn}$ and turn reversible adatom dynamics of oxidized metal species into irreversible processes of reduced metal species. Note that the different charge carriers may be also the origin of the different Tafel slopes $b$ of both manganites. For LSMO, $b \approx 60 \mathrm{mV} / \mathrm{dec}$ fits to the theoretical prediction for a chemical rate-limiting step that follows an electrochemical pre-equilibrium, probably O-O bond formation. For PCMO, the higher $b \approx 90-120 \mathrm{mV} / \mathrm{dec}$ is consistent with electron transfer as the rate-limiting step and thus may reflect the lower intrinsic electric conductivity as well as limitations due to surface reduction.

Solvated dynamic Mn adatoms and impact on reaction mechanisms of oxygen evolution. The demonstrated parallel trends in in situ ETEM and electrolysis studies underpins that the observation of enhanced surface dynamics of $\mathrm{Mn}$ adatoms in $\mathrm{H}_{2} \mathrm{O}$ is highly relevant for the real-world electrochemical behavior of the manganite electrodes. Clearly, there are differences in the properties of a Helmholtz layer in a liquid electrolyte with ion concentration of $10^{-1} \mathrm{M}$ and the thin water layer adsorbed on top of oxide materials in ETEM. In particular, the ion concentration is much lower, with typically $10^{-6}-10^{-7} \mathrm{M}$ in the vapor ${ }^{45}$ that is close to the ion concentration at neutral $\mathrm{pH}$. However, the composition of ions generated by electron impact is different since positively charged ions such as $\mathrm{H}_{2} \mathrm{O}^{+}$dominate over anions such as $\mathrm{OH}^{-}$. Our ETEM studies are performed under positive electric potentials of $\mathrm{U}=+2 \mathrm{~V}$ with respect to ground and thus under anodic polarization. Due to the high electric conductivity of the manganite lamella on highly conductive $\mathrm{Nb}$-doped $\mathrm{SrTiO}_{3}$, beam-induced potentials can be disregarded ${ }^{45}$. The comparison of hopping rates in different ambients clearly demonstrate that the observed $\geq 20 \times$ increased Mn-adatom mobility in $\mathrm{H}_{2} \mathrm{O}$ while other conditions of the experiment remain the same is not due to the electron beam. This is fully understandable from the calculation of beam-induced momentum transfer in Fig. 6. Therefore, the enhancement of thermally activated adatom motion in water is fully relevant for OER catalysis in ex situ electrochemical conditions. It can be attributed to a partial solvation of surface $\mathrm{Mn}$ in liquid $\mathrm{H}_{2} \mathrm{O}$. Such solvation effects thus do not only affect the adsorption energies of the reactants but also strongly modify the coordination and electronic properties of active sites.

The formation of a partial solvation shell of $\mathrm{H}_{2} \mathrm{O}$ around chemisorbed $\mathrm{Mn}$ adatoms in OER thus has far-reaching consequences for understanding of the reaction pathways. First 
of all, $\mathrm{Mn}$ can develop other, more flexible coordination to $\mathrm{OH}_{2}$ and $\mathrm{OH}$ compared to a static octahedral $\mathrm{Mn}-\mathrm{O}$ coordination of a frozen surface (Fig. 7). This would allow new configuration for the formation of the $\mathrm{O}-\mathrm{O}$ double bond. Furthermore, the question is raised whether the Mn dynamics affect the OER. The electron transfer rate per surface $\mathrm{Mn}$ atom derived from RRDE measurements is between $0.2 \mathrm{~s}^{-1}(1.6 \mathrm{~V})$ and $10 \mathrm{~s}^{-1}$ $(1.74 \mathrm{~V}$ vers RHE), assuming a Faraday efficiency of 1 and a complete Mn surface coverage. Due to partial coverage, these numbers represent a lower limit of the electron transfer rate per $\mathrm{Mn}$. Comparing these numbers with the observed lower limit of Mn-adatom hopping $r \approx 4 \mathrm{~s}^{-1}$, shows that both are of the same order of magnitude or even higher. Consequently, $\mathrm{Mn}$ can move over several surface sites during a full $\mathrm{O}_{2}$ evolution cycle.

In summary, our study reveals unexpectedly high $\mathrm{Mn}$-adatom hoping rates implying that the understanding of OER mechanisms requires theoretical treatment beyond the frozen surface approximation. There is strong experimental evidence from ref. ${ }^{21}$ that surface $\mathrm{Mn}$ species represent the catalytically active sites for LSMO. Fast Mn leaching at PCMO surfaces dramatically decreases OER activity. Although hopping rates and contrast of single $\mathrm{Mn}$ atoms are beyond the resolution of our experiments, the observation of dynamic multiple occupancy of surface sites by $\mathrm{Mn}$ moieties establishes a pronounced dynamical surface reconstruction of the catalyst-water interface with partial solvation of surface $\mathrm{Mn}$ atoms in $\mathrm{H}_{2} \mathrm{O}$. Mn-adatom contrast dominantly appears at interstitial surface sites rather than the nominal octahedral B sites of an unreconstructed ideal surface, suggesting a modified surface coordination. The implications of these findings are quite far-reaching. First of all, leaching of reduced transition metal species is observed for many transition metal oxides ${ }^{61,62}$. Our results imply that the underlying transition from reversible dynamics of surface atoms to irreversible leaching of surface and subsurface metal species is controlled by charge localization and a related shift of the Fermi energy. Indeed, the recent results on self-gating of electrocatalysts evidences a modification of the Fermi level at the onset of an electrochemical reaction ${ }^{63}$. The finding of pronounced reversible dynamics of partially solvated adatoms in the stationary state of OER implies that for the theoretical understanding of heterogenous catalysis some aspects of homogeneous catalysis must be taken into account, such as modified metal coordination, different valence states, solvation shell reorganization during electron transfer, solvent thermal fluctuations and possibly $\mathrm{Mn}$ displacements during intermediate formation. Incorporating these effects into theoretical analysis will open new strategies of breaking scaling relations between adsorption energies and thus new perspectives in atomic scale design of efficient and stable electrode surfaces for OER.

\section{Methods}

Film fabrication and structure. $\mathrm{La}_{0.6} \mathrm{Sr}_{0.4} \mathrm{MnO}_{3}$ and $\mathrm{Pr}_{0.33} \mathrm{Ca}_{0.67} \mathrm{MnO}_{3}$ (001) films were prepared by ion-beam sputtering (IBS) on $0.5 \mathrm{wt} \% \mathrm{Nb}$-doped $\mathrm{SrTiO}_{3}$ (STNO) substrates with (100)-orientation (CrysTec GmbH, Berlin, Germany). The $\mathrm{La}_{0.6} \mathrm{Sr}_{0.4} \mathrm{MnO}_{3}\left(\mathrm{Pr}_{0.33} \mathrm{Ca}_{0.67} \mathrm{MnO}_{3}\right)$ with thickness of $80 \mathrm{~nm}$ were grown at $800{ }^{\circ} \mathrm{C}$ $\left(650^{\circ} \mathrm{C}\right)$ in an oxygen atmosphere of $1.7 \cdot 10^{-4}$ mbar. In order to avoid rectifying junction properties for PCMO, a 20-nm thick $\mathrm{La}_{0.6} \mathrm{Sr}_{0.4} \mathrm{MnO}_{3}$ (LSMO) buffer layer was deposited without vacuum breaking. LSMO has a rhombohedral lattice, spacegroup $\mathrm{R} \overline{3} \mathrm{c}$ with a small rhombohedral tilt angle of $90.37^{\circ}$, lattice parameter $\mathrm{a}=5.47 \AA$, which converts in a pseudocubic lattice constant $\mathrm{a}_{\mathrm{c}}=3.87 \AA$. PCMO is orthorhombic, spacegroup Pbnm, with ideal lattice parameters $a=5.42 \AA, b=$ $5.45 \AA$ and $c=7.67 \AA$. Due to the larger lattice constant of STNO $(\mathrm{a}=3.92)$, the films have in-plane tensile and out-of plane compressive strain leading to $\mathrm{a}_{\mathrm{c}}=$ $3.83 \AA$ (LSMO) and c = 7.62 $\AA$ (PCMO). For LSMO, we use pseudocubic notation to facilitate the comparison of the (001) surface with the equivalent (001) surface of PCMO. The films show atomically flat surfaces with unit cell high steps. The temperature dependent electric resistivity of the films shows the expected metallike (LSMO) and small polaron hopping like (PCMO) characteristics. See Supplementary Figs. 4-7 and 24 for more details.
Backside contacts for the STNO substrate consisted of $5 \mathrm{~nm}$ Ti and $100 \mathrm{~nm} \mathrm{Pt}$ layers prepared by IBS at room temperature to ensure an ohmic contact. Carbon tape and InGa eutectic (Sigma-Aldrich, Munich, Germany, 99.99\%) served as the flexible and adjustable conductive spacer. In the final assembly, only the thin film surface is exposed to the electrolyte after fixation with chemically stable, nonconductive epoxy (Omegabond 101).

Electrochemistry. The electrochemical measurements were carried out with two Interface $1000 \mathrm{E}$ potentiostats (Gamry Instruments Inc., Warminster, PA, USA) in a bipotentiostat setup and an RRDE-3A rotator (ALS Co. Ltd., Tokyo, Japan). The rotating ring electrode consists of a disk electrode of the assembled PCMO|LSMO| NSTO electrode with a diameter of $4 \mathrm{~mm}$ and a Pt ring electrode with an inner diameter of $5 \mathrm{~mm}$ and an outer diameter of $7 \mathrm{~mm}$. All electrochemical measurements were performed in $0.1 \mathrm{M} \mathrm{KOH}$ electrolyte prepared by diluting $\mathrm{KOH}$ stock solution (Sigma-Aldrich, Munich, Germany) with deionized water (MilliQ, $>16.5 \mathrm{M} \Omega$ ). The electrolyte was saturated with $\mathrm{Ar}$ gas at least $30 \mathrm{~min}$ before measurements and continuously purged with Ar throughout the measurement. Electrode potentials were converted to the reversible hydrogen electrode (RHE) scale using $E_{\mathrm{RHE}}=\mathrm{E}_{\text {applied }}+\mathrm{E}_{\text {ref, }}$, where $\mathrm{E}_{\text {ref }}=0.993 \mathrm{~V}$ vs. SCE was obtained from the hydrogen evolution using a clean Pt disk. Cyclovoltagramms are measured at $10 \mathrm{mV} / \mathrm{s}$ with rotation of 1600 turns per minute. In Fig. 1b, between third and 4 th cycle, chronoamperometric OER is measured at 1.5, 1.55, 1.6, 1.65, 1.7, 1.75, and $1.8 \mathrm{~V}$ vs RHE, each $60 \mathrm{~s}$

Environmental TEM experiments. Transmission Electron Microscopy (TEM) experiments were carried out using a FEI Titan ETEM G2 80-300, operated at $300 \mathrm{kV}$. The microscope is equipped with a Cs-corrector of the image forming lens for enhanced high-resolution TEM (HRTEM) imaging, and a Gatan Image Filter (GIF) Quantum 965ER. A Gatan UltraScan 1000XP CCD was used to collect all movies with rate of about 4 frames per second (fps) in $512 \times 512$ pixels. The microscope was used in the high vacuum $(\mathrm{HV})$ mode $\left(\sim 10^{-5} \mathrm{~Pa}\right)$ with cold trap to reduce $\mathrm{H}_{2} \mathrm{O}$ partial pressure, as well as in environmental mode using $\mathrm{H}_{2} \mathrm{O}, \mathrm{O}_{2}$ and $\mathrm{He}$ in a pressure range between 0.01 and $100 \mathrm{~Pa}$. The following movies are provided: M01 LSMO 0.5 $\mathrm{Pa} \mathrm{H}_{2} \mathrm{O}$ flat facet, M02 LSMO 0.5 $\mathrm{Pa} \mathrm{H}_{2} \mathrm{O}$ Schwoebel Barrier, M03 LSMO HV, M04 LSMO 0.5 Pa O $\mathrm{H}_{2} \mathrm{O}, \mathrm{M} 07$ PCMO HV, M08 PCMO 0.5 $\mathrm{Pa} \mathrm{O}_{2}$, M09 LSMO $10 \mathrm{~Pa} \mathrm{O}_{2}$ recrystallization early state and M10 LSMO $10 \mathrm{~Pa} \mathrm{O}_{2}$ recrystallization late state. All time resolved HRTEM experiments were performed at a beam current of $4 \mathrm{nA}$ and beam radius between 80 and $100 \mathrm{~nm}$, resulting in a spatially in homogeneous electron dose rate of $8000-12,000 \mathrm{e} / \AA^{-2}$ s. Local electron dose rates at the location of TEM lamella surfaces are measured by calibrated CCD contrast with 0.136 electrons/ counts, yielding $\sim 10,000 \mathrm{e} / \AA^{-2} \mathrm{~s}$ for the adatom hopping studies. This dose rate is two orders of magnitude below the damage threshold in HV. Imaging over a time of $10 \mathrm{~min}$ keeps the total electron dose $\leq 610^{3} \mathrm{As} / \mathrm{cm}^{2}$, where no changes by electron irradiation are observed in HV, in good agreement with the studies of the intrinsic surface in ref. ${ }^{41}$.

Electron energy loss spectroscopy (EELS). Was performed using Gatan Quantum 965ER post-column energy filter in the same FEI Titan ETEM. Spectra of the $\mathrm{Mn} \mathrm{L}, \mathrm{O} \mathrm{K}$, and Pr M edges were acquired. For analysis of the Mn L edge spectra, Hartree-Slater (HS) type cross-section functions from Gatan's Digital Micrograph are used. The Mn valence state has been acquired from a $\mathrm{C}++$ based LabTalk script using OriginLab software. EEL spectra are extracted from spectrum images at an area of vacuum and at different $\mathrm{H}_{2} \mathrm{O}$ vapor pressure for postmortem analysis. Power-law background functions are fitted to a $50 \mathrm{eV}$ wide window before each $\mathrm{Mn}$ $\mathrm{L}$-edge and $25 \mathrm{eV}$ for $\mathrm{O}$ K-edge for background subtraction. By using dual EELS acquisition, the ZLP is used to correct the drift of the energy shift.

TEM lamella preparation. TEM lamellae were prepared from epitaxially grown (001) oriented thin films by Focused Ion-Beam lift-out technique using a noble metal free protection layer and careful low energy ion milling for the preparation of ultrathin lamella with a thickness close to the surface between 4 and $10 \mathrm{~nm}$. The loss of oxygen by Ar milling is compensated by a treatment in pure oxygen. Further details are described in the Supplementary information and Movies M09-M10.

Image simulations. Atomic models and the high-resolution TEM simulated images were calculated using the multislice method as established in the QSTEM package ${ }^{64}$, where electron optical parameters were optimized by using a metropolis algorithm implementation to fit simulated to experimental images. Structure models for the image simulations are based on the Pbnm spacegroup for PCMO with lattice parameters $a=5.42 \AA, b=5.45 \AA$ and $c=7.67 \AA$ and $\mathrm{R} 3^{\prime} c$ for LSMO using $a_{c}=3.87 \AA$. Details and parameters used for image simulation are given in the SI. 
Calculation of electron beam-induced and thermally activated hopping. Using the analytical solution given in ref. ${ }^{54}$, the adatom hopping rate is

$$
R=\frac{J}{e} \frac{\sigma_{0}}{4} F Z^{2}\left[\frac{1}{\sin ^{2}\left(\vartheta_{\min } / 2\right)}-\frac{1}{\sin ^{2}\left(\vartheta_{\max } / 2\right)}\right]
$$

where $\vartheta$ is the scattering angle of the electrons. The limits

$$
\vartheta_{\min }=\arcsin \left[2\left(\frac{E_{s}}{E_{\max }}\right)^{0.5}\right] \text { and } \vartheta_{\max }=\pi-\arcsin \left[2\left(\frac{E_{s}}{E_{\max }}\right)^{0.5}\right]
$$

are given by ratio of the captured surface energy $E_{s}$ of the adatom to the maximum possible energy transfer $E_{\max }$, which would correspond to a $180^{\circ}$ scattering, i.e.,

$$
E_{\max }(e V) \approx \frac{1.1}{A}\left[2+\frac{E_{0}}{511 k e V}\right] E_{0}(k e V)
$$

$A$ is the atomic mass, $Z$ the nuclear charge, $e$ the elementary electron charge, $J$ is the current density of the primary electron beam, $\sigma_{0}=1$ barn $=10^{-28} \mathrm{~m}^{2}$ and $F=\left(1-v^{2} / c^{2}\right)\left(\frac{v^{4}}{c^{4}}\right)$, where $c$ is the velocity of light and $v$ the velocity of the primary electrons. In order to calculate the hopping rate of an adatom on a surface, it is assumed that the kinetic energy transversal to the surface is equal to the surface activation energy $E_{a}$, i.e., $E_{s}=E_{a}$. In the Mn-adatom hopping studies, $E_{0}=$ $300 \mathrm{keV}$ and for the current density an upper limit of the experimental values of $J=2.010^{5} \mathrm{~A} / \mathrm{m}^{2}$ is chosen, corresponding to $12,000 \mathrm{e} / \AA^{2} \mathrm{~s}$.

The thermally activated hopping rate is given by

$$
R_{\mathrm{th}}=\nu_{0} e^{\frac{-E_{a}}{k_{B} T}}
$$

with adatom vibration frequency $v_{0}$ and Boltzmann constant $k_{B}$. We have chosen $v_{0}=10^{12} \mathrm{~s}^{-1}$, which for $E_{a}=0 \mathrm{eV}$ results in a thermal velocity for $\mathrm{Mn}$ atoms matching the maximum of the Maxwell-Boltzmann distribution.

\section{Data availability}

All relevant data are available from the corresponding authors on request.

Received: 8 April 2020; Accepted: 30 July 2020;

Published online: 24 September 2020

\section{References}

1. Wiechen, M., Berends, H. M. \& Kurz, P. Water oxidation catalysed by manganese compounds: from complexes to 'biomimetic rocks'. Dalton Trans. 41, 21-31 (2012)

2. McCrory, C. C. L., Jung, S., Peters, J. C. \& Jaramillo, T. F. Benchmarking heterogeneous electrocatalysts for the oxygen evolution reaction. J. Am. Chem. Soc. 135, 16977-16987 (2013).

3. Hong, W. T. et al. Toward the rational design of non-precious transition metal oxides for oxygen electrocatalysis. Energy Environ. Sci. 8, 1404-1427 (2015).

4. Lyons, M. E. G. \& Burke, L. D. Mechanism of oxygen reactions at porous oxide electrodes. Part 1.-oxygen evolution at $\mathrm{RuO}_{2}$ and $\mathrm{Ru}_{\mathrm{x}} \mathrm{Sn}_{1-\mathrm{x}} \mathrm{O}_{2}$ electrodes in alkaline solution under vigorous electrolysis conditions. J. Chem. Soc. Faraday Trans. 1 Phys. Chem. Condens. Phases 83, 299-321 (1987).

5. Bockris, J. O’M. \& Conway, B. E. Modern aspects series of chemistry. Butterworths Sci. Publ. Lond. 1, 180 (1954).

6. Conway, B. E. \& Salomon, M. Electrochemical reaction orders: applications to the hydrogen- and oxygen-evolution reactions. Electrochim. Acta 9, 1599 (1964).

7. Lodi, G., Sivieri, E., De Battisti, A. \& Trasatti, S. Ruthenium dioxide-based film electrodes-III. Effect of chemical composition and surface morphology on oxygen evolution in acid solutions. J. Appl. Electrochem. 8, 135-143 (1978).

8. Kobussen, A. G. C. \& Broers, G. H. J. The oxygen evolution on $\mathrm{La}_{0.5} \mathrm{Ba}_{0.5} \mathrm{CoO}_{3}$. Theoretical impedance behaviour for a multi-step mechanism involving two adsorbates. J. Electroanal. Chem. 126, 221-240 (1981).

9. Rossmeisl, J., Qu, Z. W., Zhu, H., Kroes, G. J. \& Nørskov, J. K. Electrolysis of water on oxide surfaces. J. Electroanal. Chem. 607, 83-89 (2007).

10. Wohlfahrt-Mehrens, M. \& Heitbaum, J. Oxygen evolution on $\mathrm{Ru}$ and $\mathrm{RuO}_{2}$ electrodes studied using isotope labelling and on-line mass spectrometry. $J$. Electroanal. Chem. 237, 251-260 (1987).

11. Grimaud, A. et al. Double perovskites as a family of highly active catalysts for oxygen evolution in alkaline solution. Nat. Commun. 4, 2439 (2013).

12. Rong, X., Parolin, J. \& Kolpak, A. M. A fundamental relationship between reaction mechanism and stability in metal oxide catalysts for oxygen evolution. ACS Catal 6, 1153-1158 (2016).

13. Mierwaldt, D. et al. In situ XANES/XPS investigation of doped manganese perovskite Catalysts. Catalysts 4, 129-145 (2014).

14. Kuan-Yu Yeh \& Michael J. Janik. In Computational Catalysis (ed. Aravind Asthagiri, M. J. J.) 116-156 (Royal Society of Chemistry, 2014).
15. Blakemore, J. D., Crabtree, R. H. \& Brudvig, G. W. Molecular catalysts for water oxidation. Chem. Rev. 115, 12974-13005 (2015).

16. Man, I. C. et al. Universality in oxygen evolution electrocatalysis on oxide surfaces. ChemCatChem 3, 1159-1165 (2011).

17. She, Z. W. et al. Combining theory and experiment in electrocatalysis: insights into materials design. Science 355, eaad4998 (2017).

18. Gono, P. \& Pasquarello, A. Oxygen evolution reaction: bifunctional mechanism breaking the linear scaling relationship. J. Chem. Phys. 152, 104712 (2020).

19. Calle-Vallejo, F., Krabbe, A. \& García-Lastra, J. M. How covalence breaks adsorption-energy scaling relations and solvation restores them. Chem. Sci. 8, 124-130 (2016)

20. Zhang, B. et al. Homogeneously dispersed multimetal oxygen-evolving catalysts. Science 352, 333-337 (2016)

21. Arrigo, R. et al. In situ study of the gas-phase electrolysis of water on platinum by NAP-XPS. Angew. Chem. Int. Ed. 52, 11660-11664 (2013).

22. Bergmann et al. Reversible amorphization and the catalytically active state of crystalline $\mathrm{Co}_{3} \mathrm{O}_{4}$ during oxygen evolution. Nat. Commun. 6, 8625 (2015).

23. Risch, M. et al. Water oxidation by amorphous cobalt-based oxides: in situ tracking of redox transitions and mode of catalysis. Energy Environ. Sci. 8, 661-674 (2015).

24. Xia, X. et al. On the role of surface diffusion in determining the shape or morphology of noble-metal nanocrystals. Proc. Natl. Acad. Sci. USA 110, 6669-6673 (2013)

25. Chen, L. Y. \& Ying, S. C. Adatoms on solid surfaces L. Phys. Rev. B 49, 838-847 (1994).

26. Shiotari, A., Okuyama, H., Hatta, S., Aruga, T. \& Hamada, I. Atomic-scale study of the formation of sodium-water complexes on $\mathrm{Cu}(110)$. Phys. Chem Chem. Phys. 20, 12210-12216 (2018).

27. Wang, Y. G., Mei, D., Glezakou, V. A., Li, J. \& Rousseau, R. Dynamic formation of single-atom catalytic active sites on ceria-supported gold nanoparticles. Nat. Commun. 6, 6511 (2015).

28. Raabe, S. et al. In situ electrochemical electron microscopy study of oxygen evolution activity of doped manganite perovskites. Adv. Funct. Mater. 22, 3378-3388 (2012).

29. Scholz, J. et al. Rotating ring-disk electrode study of oxygen evolution at a perovskite surface: Correlating activity to manganese concentration. J. Phys. Chem. C 120, 27746-27756 (2016).

30. Izgorodin, A., Winther-Jensen, O. \& MacFarlane, D. R. On the stability of water oxidation catalysts: challenges and prospects. Aust. J. Chem. 65, 638-642 (2012).

31. Kötz, R., Stucki, S., Scherson, D. \& Kolb, D. M. In-situ identification of RuO as the corrosion product during oxygen evolution on ruthenium in acid media. J. Electroanal. Chem. 172, 211-219 (1984).

32. da Silva, G. C., Mayrhofer, K. J. J., Ticianelli, E. A. \& Cherevko, S. Dissolution stability: the major challenge in the regenerative fuel cells bifunctional catalysis. J. Electrochem. Soc. 165, F1376-F1384 (2018).

33. Stoerzinger, K. A. et al. The role of Ru redox in $\mathrm{pH}$-dependent oxygen evolution on rutile ruthenium dioxide. Surfaces. Chem 2, 668-675 (2017)

34. Cherevko, S. Stability and dissolution of electrocatalysts: building the bridge between model and "real world" systems. Curr. Opin. Electrochem. 8, 118-125 (2018).

35. Kanan, M. W. \& Nocera, D. G. In situ formation of an oxygen-evolving catalyst in neutral water containing phosphate and $\mathrm{Co}^{2+}$. Science 321, 1072-1075 (2008)

36. Handoko, A. D., Wei, F., Jenndy, Yeo, B. S. \& Seh, Z. W. Understanding heterogeneous electrocatalytic carbon dioxide reduction through operando techniques. Nat. Catal. 1, 922-934 (2018).

37. Kasian, O., Grote, J. P., Geiger, S., Cherevko, S. \& Mayrhofer, K. J. J. The common intermediates of oxygen evolution and dissolution reactions during water electrolysis on iridium. Angew. Chem. Int. Ed. 57, 2488-2491 (2018).

38. Yoshida, H. et al. Visualizing gas molecules interacting with supported nanoparticulate catalysts at reaction conditions. Science 335, 317-319 (2012).

39. Lin, Y., Wu, Z., Wen, J., Poeppelmeier, K. R. \& Marks, L. D. Imaging the atomic surface structures of $\mathrm{CeO}_{2}$ nanoparticles. Nano Lett. 14, 191-196 (2014).

40. Bugnet, M., Overbury, S. H., Wu, Z. L. \& Epicier, T. Direct visualization and control of atomic mobility at $\{100\}$ surfaces of Ceria in the environmental transmission electron microscope. Nano Lett. 17, 7652-7658 (2017).

41. Kuwauchi, Y., Yoshida, H., Akita, T., Haruta, M. \& Takeda, S. Intrinsic catalytic structure of gold nanoparticles supported on $\mathrm{TiO}_{2}$. Angew. Chem. Int. Ed. 51, 7729-7733 (2012)

42. Roddatis, V., Lole, G. \& Jooss, C. In situ preparation of $\operatorname{Pr}_{1-\mathrm{x}} \mathrm{Ca}_{\mathrm{x}} \mathrm{MnO}_{3}$ and $\mathrm{La}_{1-\mathrm{x}} \mathrm{Sr}_{\mathrm{x}} \mathrm{MnO}_{3}$ catalysts surface for high-resolution environmental transmission electron microscopy. Catalysts 9, 751 (2019).

43. Möbus, G. et al. Dynamics of polar surfaces on ceria nanoparticles observed in situ with single-atom resolution. Adv. Funct. Mater. 21, 1971-1976 (2011). 
44. Yuan, W. et al. Visualizing $\mathrm{H}_{2} \mathrm{O}$ molecules reacting at $\mathrm{TiO}_{2}$ active sites with transmission electron microscopy. Science 367, 428-430 (2020).

45. Mildner, S. et al. Environmental TEM study of electron beam induced electrochemistry of $\mathrm{Pr}_{0.64} \mathrm{Ca}_{0.36} \mathrm{MnO}_{3}$ catalysts for oxygen evolution. J. Phys. Chem. C 119, 5301-5310 (2015).

46. Bakardjieva, S., Bezdička, P., Grygar, T. \& Vorm, P. Reductive dissolution of microparticulate manganese oxides. J. Solid State Electrochem. 4, 306 (2000).

47. Nemudry, A., Rudolf, P. \& Schöllhorn, R. Topotactic electrochemical redox reactions of the defect perovskite $\mathrm{SrCoO}_{2.5+\mathrm{x}}$. Chem. Mater. 8, 2232 (1996)

48. Köhler, L., Szabadics, L., Jooss, C. \& Risch, M. Peroxide yield of the (001) $\mathrm{La}_{0.6} \mathrm{Sr}_{0.4} \mathrm{MnO}_{3}$ surface as a bifunctional electrocatalyst for the oxygen reduction reaction and oxygen evolution reaction in alkaline media. Batter. Supercaps 2, 364-372 (2019).

49. Stoerzinger, K. A., Qiao, L., Biegalski, M. D. \& Shao-Horn, Y. Correction to "Orientation-Dependent Oxygen Evolution Activities of Rutile $\mathrm{IrO}_{2}$ and $\mathrm{RuO}_{2}$ ". J. Phys. Chem. Lett. 5, 1636-1641 (2015).

50. Ketteler, G. et al. The nature of water nucleation sites on $\mathrm{TiO}_{2}(110)$ surfaces revealed by ambient pressure X-ray photoelectron spectroscopy. J. Phys. Chem. C 111, 8278-8282 (2007).

51. Srinivasan, S. G. et al. Crystal structures, surface stability, and water adsorption energies of La-Bastnäsite via density functional theory and experimental studies. J. Phys. Chem. C 120, 16767-16781 (2016).

52. Opitz, A., Scherge, M., Ahmed, S. I. U. \& Schaefer, J. A. A comparative investigation of thickness measurements of ultra-thin water films by scanning probe techniques. J. Appl. Phys. 101, 064310 (2007).

53. Drazin, J. W. \& Castro, R. H. R. Water adsorption microcalorimetry model: deciphering surface energies and water chemical potentials of nanocrystalline oxides. J. Phys. Chem. C 118, 10131-10142 (2014).

54. Egerton, R. F. Beam-induced motion of adatoms in the transmission electron microscope. Microsc. Microanal. 19, 479-486 (2013).

55. Scholz, J. et al. Tailoring the oxygen evolution activity and stability using defect chemistry. Catalysts 7, 139 (2017).

56. Hartinger, C., Mayr, F., Loidl, A. \& Kopp, T. Polaronic excitations in colossal magnetoresistance manganite films. Phys. Rev. B Condens. Matter Mater. Phys. 73, 024408 (2006).

57. Hoffmann, J. et al. Effects of interaction and disorder on polarons in colossal resistance manganite $\mathrm{Pr}_{0.68} \mathrm{Ca}_{0.32} \mathrm{MnO}_{3}$ thin films. Mater. Res. Express $\mathbf{1}$, 046403 (2015).

58. Daoud-Aladine, A., Rodríguez-Carvajal, J., Pinsard-Gaudart, L., FernándezDíaz, M. T. \& Revcolevschi, A. Zener polaron ordering in half-doped manganites. Phys. Rev. Lett. 89, 097205 (2002).

59. Jooss, C. et al. Polaron melting and ordering as key mechanisms for colossal resistance effects in manganites. Proc. Natl Acad. Sci. USA 104, 13597 (2007).

60. Sotoudeh, M. et al. Electronic structure of $\mathrm{Pr}_{1-\mathrm{x}} \mathrm{Ca}_{\mathrm{x}} \mathrm{MnO}_{3}$. Phys. Rev. B 95, 235150 (2017)

61. Najafpour, M. M., Sedigh, D. J., Pashaei, B. \& Nayeri, S. Water oxidation by nano-layered manganese oxides in the presence of cerium(iv) ammonium nitrate: important factors and a proposed self-repair mechanism. N. J. Chem. 37, 2448 (2013).

62. Chivot, J., Mendoza, L., Mansour, C., Pauporté, T. \& Cassir, M. New insight in the behaviour of $\mathrm{Co}-\mathrm{H}_{2} \mathrm{O}$ system at $25-150{ }^{\circ} \mathrm{C}$, based on revised Pourbaix diagrams. Corros. Sci. 50, 62-69 (2008)

63. He, Y. et al. Self-gating in semiconductor electrocatalysis. Nat. Mater. 18, 1098-1104 (2019).

64. Koch, C. Determination of Core Structure Periodicity and Point Defect Along Dislocations. Ph.D. Thesis, Arizona State University (2002).
65. Palcut, M., Wiik, K. \& Grande, T. Cation self-diffusion and nonstoichiometry of lanthanum manganite studied by diffusion couple measurements. J. Phys. Chem. C 111, 813-822 (2007)

66. Polfus, J. M., Yildiz, B. \& Tuller, H. L. Origin of fast oxide ion diffusion along grain boundaries in Sr-doped $\mathrm{LaMnO}_{3}$. Phys. Chem. Chem. Phys. 20, 19142-19150 (2018).

67. Miyoshi, S. \& Martin, M. B-Site cation diffusivity of $\mathrm{Mn}$ and $\mathrm{Cr}$ in perovskitetype $\mathrm{LaMnO}_{3}$ with cation-deficit nonstoichiometry. Phys. Chem. Chem. Phys. 11, 3063 (2009).

\section{Acknowledgements}

This work was financially supported by the DFG SFB 1073, projects C02, C03 and B02 and use of equipment in the "Collaborative Laboratory and User Facility for Electron Microscopy" (CLUE) at University of Goettingen are gratefully acknowledged. We thank Ch. Luderer and D. Busse for post electrochemical TEM analysis of LSMO films.

\section{Author contributions}

G.L. and V.R. carried out the ETEM experiments. T.M. and U.R contributed to image analysis. J.G. and G.W. did manganite epitaxy and structural analysis as well as electrochemical measurements. M.R. contributed to analysis of electrochemical data. L.R. provided software for image simulation. P.B. contributed to theoretical analysis of the results. C.J. did calculations on beam effects and water adsorption and wrote the manuscript. All authors commented on, discussed and edited the manuscript.

\section{Competing interests}

The authors declare no competing interests.

\section{Additional information}

Supplementary information is available for this paper at https://doi.org/10.1038/s43246020-00070-6.

Correspondence and requests for materials should be addressed to C.J.

Reprints and permission information is available at http://www.nature.com/reprints

Publisher's note Springer Nature remains neutral with regard to jurisdictional claims in published maps and institutional affiliations.

(c) Open Access This article is licensed under a Creative Commons Attribution 4.0 International License, which permits use, sharing, adaptation, distribution and reproduction in any medium or format, as long as you give appropriate credit to the original author(s) and the source, provide a link to the Creative Commons license, and indicate if changes were made. The images or other third party material in this article are included in the article's Creative Commons license, unless indicated otherwise in a credit line to the material. If material is not included in the article's Creative Commons license and your intended use is not permitted by statutory regulation or exceeds the permitted use, you will need to obtain permission directly from the copyright holder. To view a copy of this license, visit http://creativecommons.org/ licenses/by/4.0/.

(c) The Author(s) 2020 